\title{
A modified grey wolf optimizer for improving wind plant energy production
}

\author{
Mohd Zaidi Mohd Tumari ${ }^{1}$, Mohd Helmi Suid ${ }^{2}$, Mohd Ashraf Ahmad ${ }^{3}$ \\ ${ }^{1}$ Faculty of Engineering Technology, UniversitiTeknikal Malaysia Melaka (UTEM), Malaysia \\ ${ }^{2,3}$ Faculty of Electrical and Electronics Engineering, University Malaysia Pahang (UMP), Malaysia
}

\begin{tabular}{l} 
Article Info \\
\hline Article history: \\
Received Aug 10, 2019 \\
Revised Nov 30, 2019 \\
Accepted Dec 14, 2019 \\
\hline
\end{tabular}

Keywords:

Energy production

Metaheuristic algorithm

Nature inspired algorithm

Wake interactions

Wind farm

\begin{abstract}
The main problem of existing wind plant nowadays is that the optimum controller of single turbine degrades the total energy production of wind farm when it is located in a large wind plant. This is owing to its greedy control policy that can not cope with turbulence effect between turbines. This paper proposes a Modified Grey Wolf Optimizer (M-GWO) to improvise the controller parameter of an array of turbines such that the total energy production of wind plant is increased. The modification employed to the original GWO is in terms of the updated mechanism. This modification is expected to improve the variation of exploration and exploitation rates while avoiding the premature convergence condition. The effectiveness of the MGWO is applied to maximize energy production of a row of ten turbines. The model of the wind plant is derived based on the real Horns Rev wind plant in Denmark. The statistical performance analysis shows that the MGWO provides the highest total energy production as compared to the standard GWO, Particle Swarm Optimization (PSO) and Safe Experimentation Dynamics (SED) methods.
\end{abstract}

Copyright $@ 2020$ Institute of Advanced Engineering and Science. All rights reserved.

\section{Corresponding Author:}

Mohd Zaidi Mohd Tumari,

Faculty of Engineering Technology,

Jln Hang Tuah Jaya, 76100, Melaka.

Email: mohdzaidi.tumari@utem.edu.my

\section{INTRODUCTION}

One of the primary issues of the wind plant research nowadays is that the optimal controller parameters of individual turbine do not guarantee an optimal energy production of the whole wind plant. This is due to the wake interaction between turbines in the wind plant that degrades the total energy production of the wind plant. Therefore, it is necessary to fine tune again the optimal controller parameters of each wind turbine in the wind plant. These controller parameters can be considered as angle of blade and yaw or/and torque generator. Unlike the problem of finding the control parameters of single turbine, which normally solved using the model-based strategy, the problem of optimizing of controller parameters of an array of turbines in wind plant is more challenging task. This is due to the difficulty in obtaining the dynamic behavior of the wake interactions or turbulence between turbines, which is chaotic in nature and depended on time varying wind speed magnitude with different directions. Hence, it is almost impossible to find accurate control parameters of the wind plant using the model-based control strategy.

So far, there are several of strategies that have been proposed to find the optimal controller parameters of an array of turbines in the wind plant. One of the recent popular strategies is based on the datadriven method that totally relies on the total energy production data of wind plant. These include Safe Experimentation Dynamics (SED) [1-2] and cooperative static control [3]. Those methods are under the class of game theoretic method that defines the controller parameter of each turbine as a player to achieve the given goal which is the total energy production. In [4-5], the researchers propose a Maximum energy Point 
Tracking (MPPT) method by incorporating a time varying energy production measurement of wind plant. Here, they show that the proposed MPPT method provides more fast convergence of total energy production as compared to the method in [2]. Similarly, the works in [6] also considered the same model of wind plant as in [5]. Here, by manipulating the structure of the given wind plant, the proposed Multi-Resolution Simultaneous Perturbation Stochastic Approximation (MR-SPSA) is able to produce faster convergence and higher total energy production than the strategy in [5]. In [7], they have shown that a basic Random Search method can provide better total energy production than SED method. Note that, the works in [1-7] are based on the single agent based optimization methods. Meanwhile, the first population based optimization method that is applied to this problem is reported in [8]. Here, they show that the Spiral Dynamics Algorithm (SDA) provides better total energy production than the Particle Swarm Optimization (PSO) and SED methods. Similarly, in [9], it is claimed that the Moth Flame Optimization (MFO) exhibits better energy production than the SDA method in [8].

On the other hand, a Grey Wolf Optimizer (GWO) [10], which is in the class of nature inspired based optimization, can be also considered as a potential tool for improving energy production of wind plant. This is because the GWO has been successfully solved various types of real world problems, such as optimal reactive power coordination [11], vehicle engine connecting rod [12], unmanned aerial vehicles $[13,14]$, facial image super-resolution [15, 16], surface waves estimation [17], PID controller tuning [18, 19]. The essential feature of the GWO algorithm is that it inspires the hierarchy, leadership and hunting techniques of grey wolves. GWO adopted four types of grey wolves, which are alpha, beta, delta, and omega that play important roles in hunting, searching, encircling, and attacking. However, based on our preliminary works using a row of ten turbines, the standard GWO is still not able to provide high accuracy of total energy production. Therefore, it motivates us to modify the standard GWO algorithm such that a better total energy production of wind plant can be achieved.

This paper proposes a Modified Grey Wolf Optimizer (M-GWO) to improve energy production of wind plant. In particular, an existing linear updated mechanism in the standard GWO is modified to a new nonlinear updated mechanism. This modification is necessary to provide an optimal portion of exploration and exploitation for wind plant optimization problem. The proposed nonlinear updated mechanism is based on our study in [20], which has been employed to Sine Cosine Algorithm. In [20], it is shown that the proposed updated mechanism has significantly improved the optimization accuracy of several benchmark functions.

Furthermore, a single row wind plant with ten turbines is used to assess the effectiveness of the MGWO. Here, the mathematical model of the wind plant is adopted from the Park model [21]. The investigation on the optimum combination of the maximum number of iterations and the number of agents is also considered in this wind plant problem. Since the M-GWO is in the type of multi-agent based method, a large number of trials are considered to evaluate its consistency in producing high accuracy of total energy production. The statistical performance analysis of the wind plant total energy production using the proposed method is shown. In addition, a comparative assessment between the M-GWO, the standard GWO, the PSO and the SED [2] approaches is presented.

\section{PROBLEM FRAMEWORK}

Consider the energy production of turbine $\mathrm{k}$ is defined by $E_{k}\left(h_{1}, h_{2}, \ldots, h_{m}\right)(k=1,2, \ldots, m)$, where $m$ is the total number of turbines in the wind plant and $h_{k}(k=1,2, \ldots, m)$ is the controller parameter of each turbine $k$. Naturally, the incoming wind speed to a wind plant can be considered in various angle of directions with random position of turbines. Hence, the controller parameters of other turbines $h_{1}, h_{2}, \ldots, h_{k-1}, h_{k+1}, \ldots$, $h_{m}$, which is not include controller parameter of turbine $k$, would also influence the energy production $E_{k}$ of turbine $k$ due to the wake interactions between turbines. Equivalently, the changes of $h_{k}$ might also influence the energy productions of other turbines $E_{1}, E_{2}, \ldots ., E_{k-1}, E_{k+1}, \ldots, E_{m}$. The exact formulation of function $E_{k}\left(h_{1}\right.$, $h_{2}, \ldots, h_{m}$ ) can be negligible in this study due to highly complex turbulence interactions among turbines, which are too problematic to obtain its mathematical model. Nevertheless, it can be assumed that the energy production data of each turbine is measureable. Thence, the total measured energy production can be written as follows:

$$
\bar{E}\left(h_{1}, h_{2}, \ldots, h_{m}\right)=\sum_{k=1}^{m} E_{k}\left(h_{1}, h_{2}, \ldots, h_{m}\right)
$$

Finally, this wind plant data-driven control problem can be stated as: 
Problem 2.1: Find the controller parameter of each turbine $h_{k}(k=1,2, \ldots, m)$ such that the total energy production $\bar{E}\left(h_{1}, h_{2}, \ldots, h_{m}\right)$ in (1) is maximized without any knowledge on the relation between $h_{k}(k=1,2$, $\ldots, m)$ and $\bar{E}$.

\section{MODIFIED GREY WOLF OPTIMIZER}

In this section, the proposed M-GWO for optimizing controller parameters of wind turbines in wind plant is explained. Firstly, a standard Grey Wolf Optimizer (GWO) is briefly described. This is followed by the description of the modification in GWO, which is able to provide better total energy production of wind plant.

\subsection{Summary of the Standard GWO}

A brief description of the standard GWO, which is introduced in [10], is shown. Consider $g: \boldsymbol{R}^{n} \rightarrow \boldsymbol{R}$ is the cost function, $\boldsymbol{v}_{i}(i=1,2, \ldots, N)$ is the design variable and $N$ is the number of agents. Let $v_{i j}$ $(j=1,2, \ldots, n)$ is $j$-th element of the vector $\boldsymbol{v}_{i}$. Thence, a maximization problem is expressed as:

$$
\arg \max _{v_{i}(1), v_{i}(2), . .} g_{i}\left(v_{i}(t)\right)
$$

for iteration $t=1,2, \ldots$ and for each agent $i$. The GWO algorithm updates the design variable vector for each agent iteratively as:

$$
v_{i}(t+1)=\frac{v^{1}+v^{2}+v^{3}}{3}
$$

Where

$$
\boldsymbol{v}^{1}=\boldsymbol{v}^{\alpha}-\boldsymbol{A} \cdot\left|\boldsymbol{C} \cdot \boldsymbol{v}^{\alpha}-\boldsymbol{v}_{i}(t)\right|, \boldsymbol{v}^{2}=\boldsymbol{v}^{\beta}-\boldsymbol{A} \cdot\left|\boldsymbol{C} \cdot \boldsymbol{v}^{\beta}-\boldsymbol{v}_{i}(t)\right|, \boldsymbol{v}^{3}=\boldsymbol{v}^{\delta}-\boldsymbol{A} \cdot\left|\boldsymbol{C} \cdot \boldsymbol{v}^{\delta}-\boldsymbol{v}_{i}(t)\right|
$$

for $i=1,2, \ldots, N$. In (4), the vectors $\boldsymbol{A}$ and $\boldsymbol{C}$ are expressed as follows:

$$
\begin{aligned}
& \boldsymbol{A}=2 \boldsymbol{a} \cdot \boldsymbol{r}_{1}-\boldsymbol{a}, \\
& \boldsymbol{C}=2 \cdot \boldsymbol{r}_{2},
\end{aligned}
$$

where $\boldsymbol{r}_{1}$ and $\boldsymbol{r}_{2}$ are the random vectors, where each element is generated independently from 0 to 1 , and each element of $\boldsymbol{a}$ is linearly decreased from 2 to 0 over the course of iteration using the following equation:

$$
a=2\left(1-\frac{t}{T}\right),
$$

where $T$ is the maximum number of iterations. Note that the vectors of $\boldsymbol{A}$ and $\boldsymbol{C}$ in (4), which consists of random vectors $\boldsymbol{r}_{1}$ and $\boldsymbol{r}_{2}$, are generated independently between $\boldsymbol{v}^{1}, \boldsymbol{v}^{2}$ and $\boldsymbol{v}^{3}$. Furthermore, the best design variable solution in (4) is denoted by $\boldsymbol{v}^{\alpha}$, followed by $\boldsymbol{v}^{\beta}$ and $\boldsymbol{v}^{\delta}$, which determine that they have better knowledge of optimal solution than other agents.

\subsection{Modified GWO (M-GWO)}

The modification of the standard GWO algorithm is explained in this section. In the original version of GWO, the value of $a$ in (7) is linearly decayed from 2 to 0 during the tuning process, which is claimed to provide an exact balance between exploration and exploitation. Nevertheless, the setting in (7) maybe limited to several applications only, while it is good to propose more generic equation of $a$ that can cover more general class of applications. Motivated from the above limitation, (7) is modified to produce a new generic equation of updated step size as follows:

$$
\tilde{a}=\sigma\left(1-\left(\frac{t}{T}\right)^{\mu}\right)^{\lambda}
$$


Which is directly adopted from our previous work in [20]. In (8), the symbols $\sigma, \mu$ and $\lambda$ are the positive constant values that are introduced to regulate the portion of exploration and exploitation during the tuning process. Although there are several researchers that proposed exponential curve [22] and nonlinear modulation index [23] in the original $a$, their equations are limited to only one curve, while (8) can generate two curves during the whole iterations. As a result, it is expected that our new $\tilde{a}$ can provide more choices of exploration and exploitation portions as compared to the exponential and nonlinear modulation index versions. Finally, the M-GWO will follow the same procedure of GWO by replacing (7) with (8).

\subsection{Applications of M-GWO for Improving Energy Production of Wind Farm}

The procedure to apply the proposed M-GWO for improving energy production of wind plant is shown in this section. By applying the M-GWO in the previous section, the procedure for data-driven control of wind plant is given as follows:

Step 1: Select the values of $\sigma, \mu$ and $\lambda$ in (8).

Step 2: Execute the M-GWO by setting $\bar{E}=g_{i}$ and $h_{k}(k=1,2, \ldots, m)=v_{i j}(j=1,2, \ldots, n)$ for each $i$.

Step 3: The algorithm stops with the solution $h_{k}{ }^{*}(k=1,2, \ldots, m)=v^{\alpha}{ }_{j}(j=1,2, \ldots, n)$ after $T$ iterations and the corresponding total energy production $\bar{E}\left(h_{1}{ }^{*}, h_{2}{ }^{*}, \ldots, h_{m}{ }^{*}\right)$ is observed. Note that $v_{j}^{\alpha}$ is $j$ th element of the vector $\boldsymbol{v}^{\alpha}$.

The flow of the data-driven wind plant control scheme based on M-GWO is illustrated in Figure 1. The detail of the pseudo-code can be downloaded from the following link: http://bit.do/m_gwo

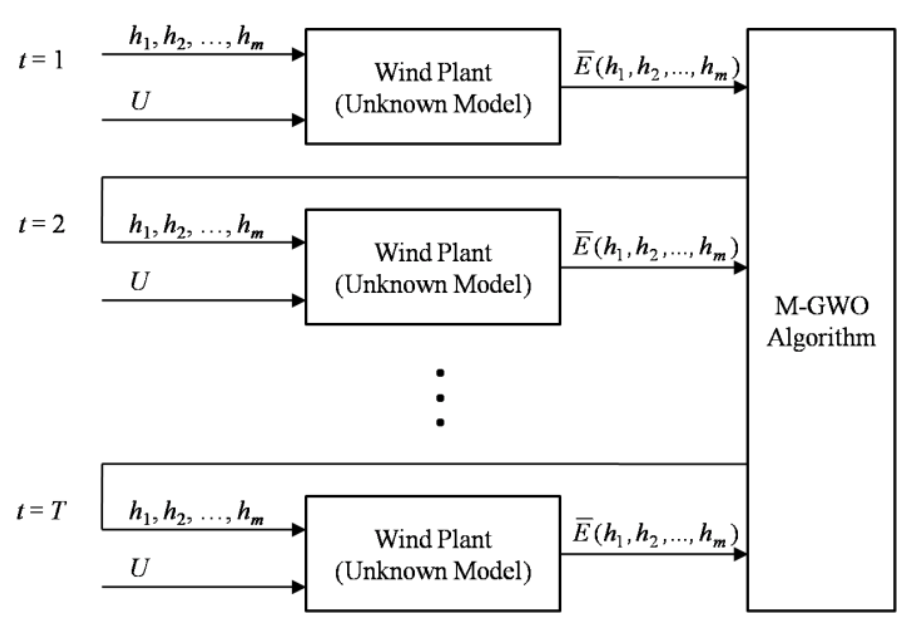

Figure 1. The structure of data-driven wind plant control using M-GWO

\section{NUMERICAL RESULTS AND DISCUSSION}

The effectiveness of the M-GWO based method for improving energy production of wind plant is evaluated in this section. Initially, the model of the wind plant introduced by [21] is explained. Then, the MGWO is applied to the developed wind plant model.

\subsection{Wind Plant Mathematical Model}

Consider the energy production of each turbine $k$ is described as

$$
E_{k}=2 \rho A_{k} h_{k}\left(1-h_{k}\right)^{2} W_{k}
$$

where $\rho$ is the air density, $A_{k}$ is the rotor swept area of turbine $k$ and $W_{k}$ is the accumulation wind speed produced by the upstream wind turbines. The expression of $W_{k}$ is given by

$$
W_{k}=U\left(1-2 \sqrt{\sum_{l=1}^{F}\left(h_{l}\left(\frac{D_{l}}{D_{l}+2 \varphi d_{l k}}\right)^{2} \frac{A_{o v}}{A_{k}}\right)^{2}}\right)
$$


where $U$ is the incoming wind speed in front of the first turbine, $F$ is the number of upstream turbine, $l$ is the index to represent the upstream turbine, $D_{l}$ is the diameter of turbine rotor, $\varphi$ is the parameter to represent the gradient of the wind turbulence between two turbines, $d_{l k}$ is the distance between turbine $k$ and upstream turbine $l, A_{o v}$ is the overlap area of turbulence from the upstream turbine.

Remarks: Note that in this study, the proposed M-GWO only requires the data of total energy production of wind plant $\bar{E}$ without even know the exact relation between $\bar{E}\left(h_{1}, h_{2}, \ldots, h_{m}\right)$ and $h_{k}(k=1,2, \ldots, m)$ as expressed in (9) and (10). Therefore, this problem can be treated as a data-driven or model-free control problem. In other words, the proposed data-driven based M-GWO can be also applied in real wind plant optimization problem since it only requires the data of total energy production only.

\subsection{Example of Single Row Wind Plant with 10 Turbines}

The effectiveness of the M-GWO is observed in this section. The performance of M-GWO is verified on a 10 turbines row wind plant as presented in Figure 2. The wind plant model in the previous section is adopted for ten turbines $(m=10)$ with $80 \mathrm{~m}$ of turbine rotor diameter on each. The location of wind turbine is positioned in a row with a same $560 \mathrm{~m}$ distance between each turbine. The values of air density and the turbulence gradient parameters are $\rho=1.225 \mathrm{~kg} / \mathrm{m} 3$ and $\varphi=0.04$, respectively. In order to experience large turbulence effect, it is assumed that the wind direction is 90 degree to the rotor swept area with a fix incoming wind speed at $U=8 \mathrm{~m} / \mathrm{s}$.

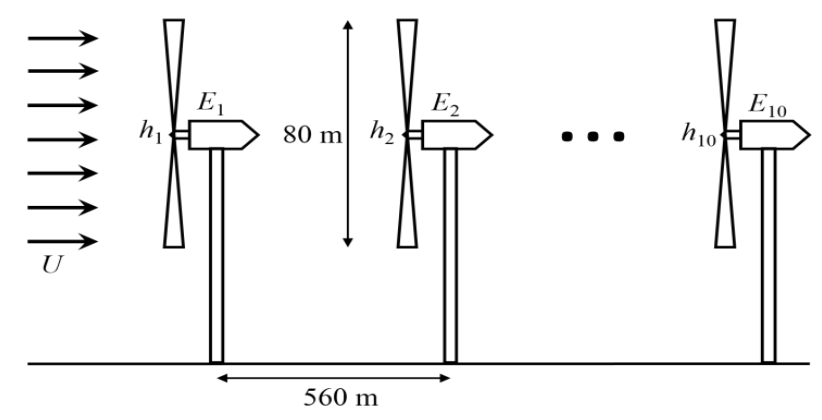

Figure 2. The location of 10 turbines in wind plant

In this work, a comparative assessment between the proposed M-GWO and the standard GWO, PSO and SED in [2] in terms of maximum total energy production is considered. The optimum combination of number of agents and maximum number of iterations are $N=10$ and $T=1000$ that contributes to 10,000 number of function evaluations. The coefficients of M-GWO is set as $\mu=0.008, \lambda=1$ and $\sigma=1.7$, after performed several initial investigations. Similarly, the combination of $N$ and $T$ is set to be the same as MGWO. The coefficients of PSO is fixed as $c_{0}=0.9, c_{1}=0.1$, and $c_{2}=0.5$, with $N=20$ and $T=500$, to produce the same number of function evaluations. Please see $[24,25]$ for the details of the PSO algorithm. Meanwhile, the SED with interval step size $K_{G}=0.03$ and the probability of changing the design variable $E=$ 0.3 are adopted. Since the SED is a single agent based optimization method, the number of function evaluations is set to be the same as maximum number of iterations, which is $T=10,000$. Please see [2] for the details of SED algorithm. The initial control parameter of each turbine for M-GWO, GWO and PSO are set randomly between ranges of $[0,1 / 3]$, while the initial control parameter of SED is set at $1 / 3$ for all turbines. Note that the value of $1 / 3$ is considered as the optimum controller parameter of individual turbine produced by the designer before it is placed in the wind plant. Here, 100 trials are carried out for executing the M-GWO, GWO, PSO and SED, in order to observe the performance of each method due to the randomization effect.

Table 1 tabulates the statistical performance of the total energy production for 10,000 number of function evaluations. In particular, the performances of the wind plant total energy production are assessed based on its mean, best, worst and standard deviations, after 100 independent trials. It can be seen that the MGWO yields the highest best total energy production with the value of $4.7648415724 \mathrm{MW}$, followed by the PSO (4.7648415723 MW) and SED (4.7648415242 MW) and the GWO (4.7648412912 MW). A similar trend can be observed for the worst and mean values of the total energy production. Furthermore, the proposed M-GWO also produces slightly lower value of standard deviation than the GWO, PSO and SED. The finding justifies that the proposed M-GWO is robust to the stochastic effect while consistently 
improving total energy production. On the other hand, based on the obtained optimal controller parameters, the best optimal controller parameters of the M-GWO are recorded as $h_{k}^{*}(k=1,2, \ldots, 10)=(0.2061,0.1611$, $0.1648,0.1651,0.1698,0.1173,0.2258,0.1877,0.1837,0.3333)$. It shows that the optimal controller parameter value of the first turbine is larger compared to the values of middle turbines, but still less than the $1 / 3$. However, the value in the final turbine is maintained as the initial controller parameter, which is $1 / 3$. It shows that the optimum values of the controller parameters of the upstream wind turbines are lower than the $1 / 3$ to reduce the turbulence effect and increase the accumulation wind speed to the downstream turbines. In contrast, since there is no more downstream turbine for the final turbine, its controller parameter is fixed at the full capacity of $1 / 3$. This trend is similar to existing investigation on data-driven control of wind plant, e.g., [2], while improving the total energy production.

Table 1. Comparative Assessment of Total Energy Production between M-GWO, GWO, PSO and SED

\begin{tabular}{ccccc}
\hline Statistical results & M-GWO & GWO & PSO & SED [2] \\
\hline Mean (MW) & $\mathbf{4 . 7 6 4 8 4 1 5 7 2 3}$ & 4.7648390511 & 4.7648415625 & 4.7644075485 \\
Best (MW) & $\mathbf{4 . 7 6 4 8 4 1 5 7 2 4}$ & 4.7648412912 & 4.7648415723 & 4.7648415242 \\
Worst (MW) & $\mathbf{4 . 7 6 4 8 4 1 5 7 2 0}$ & 4.7648339341 & 4.7648414855 & 4.7627457259 \\
Standard Deviation & $\mathbf{6 . 6 7 8} \times \mathbf{1 0}^{-5}$ & 1.3615 & 0.0141665007 & $4.513106 \times 10^{2}$ \\
\hline
\end{tabular}

\section{CONCLUSION}

In this study, a new Modified Grey Wolf Optimizer (M-GWO) for improving wind plant control parameters has been presented. Here, the nonlinear updated step size is proposed in GWO such that the user can freely control the portion of exploration and exploitation based in specific applications. The proposed MGWO is verified on a single row wind plant of 10 turbines, which is based on the Horn Rew wind plant model in Denmark. The results prove that proposed modifications on the original GWO have significantly improved the total energy production of wind plant. In particular, the M-GWO has produced a slightly higher total energy production than the original GWO, PSO and SED in terms of mean, best, worst and standard deviation. Thence, it justifies the potential of M-GWO for data-driven control of wind plant.

\section{ACKNOWLEDGEMENTS} RDU1703151.

The work was fully supported by University Malaysia Pahang under the research grant

\section{REFERENCES}

[1] J.R. Marden, S.D. Ruben, L.Y. Pao, “Surveying game theoretic approaches for wind farm optimization," Proc. of the AIAA Aerospace Sciences Meeting, pp. 1-10, 2012.

[2] J.R. Marden, S.D. Ruben, L.Y. Pao, "A model-free approach to wind farm control using game theoretic methods," IEEE Transactions on Control Systems Technology, Vol. 21(4), pp. 1207-1214, 2013.

[3] J. Park, K.H. Law, "A data-driven, cooperative wind farm control to maximize the total power production," Applied Energy, Vol. 165, pp. 151-165, 2016.

[4] P.M. Gebraad, F.C. van Dam, J.W. van Wingerden, "A model-free distributed approach for wind plant control," Proc. American Control Conference, pp. 628-633, 2013.

[5] P.M. Gebraad, J.W. van Wingerden, "Maximum power-point tracking control for wind farms," Wind Energy, Vol. 18(3), pp. 429-447, 2015.

[6] M.A. Ahmad, S.I. Azuma, T. Sugie, "A model-free approach for maximizing power production of wind farm using multi-resolution simultaneous perturbation stochastic approximation,” Energies, Vol. 7(9), pp. 5624-5646, 2014.

[7] M.A. Ahmad, M.R. Hao, R.M.T. Raja Ismail, A.N.K. Nasir, "Model-free wind farm control based on random search," Proc. of IEEE International Conference on Automatic Control and Intelligent Systems, pp. 131-134, 2016.

[8] M.R. Hao, R.M.T. Raja Ismail, M.A. Ahmad, “Using Spiral Dynamic Algorithm for Maximizing Power Production of Wind Farm," Proc. of IEEE International Conference on Applied System Innovation, pp. 1706-1709, 2017.

[9] M.A. Md Idris, M.R. Hao, M.A. Ahmad, "A Data Driven Approach to Wind Plant Control using Moth-Flame Optimization (MFO) Algorithm," International Journal on Advanced Science, Engineering and Information Technology, pp. 18-23, 2019.

[10] S. Mirjalili, S.M. Mirjalili, A. Lewis, "Grey wolf optimizer”, Advances in engineering software, Vol. 69, pp. 46-61, 2014.

[11] M. Sankaraiah, S.S. Reddy, M.V. Kumar, "GWO Based Optimal Reactive Power Coordination of DFIG, ULTC and Capacitors”, Indonesian Journal of Electrical Engineering and Computer Science, Vol. 11(3), pp. 805-813, 2018.

[12] B.S. Y1ld1z, A.R. Y1ld1z, "Comparison of grey wolf, whale, water cycle, ant lion and sine-cosine algorithms for the optimization of a vehicle engine connecting rod," Materials Testing, Vol. 60(3), pp. 311-315, 2018. 
[13] M. Radmanesh, M. Kumar, M. Sarim, "Grey Wolf optimization based sense and avoid algorithm in a Bayesian framework for multiple UAV path planning in an uncertain environment," Aerospace Science and Technology, Vol. 77, pp. 168-179, 2018 .

[14] S. Zhang, Y. Zhou, Z. Li, W. Pan, "Grey wolf optimizer for unmanned combat aerial vehicle path planning", Advances in Engineering Software, Vol. 99, pp. 121-136, 2016.

[15] S.S. Rajput, V.K. Bohat, K.V. Arya, "Grey wolf optimization algorithm for facial image super-resolution," Applied Intelligence, pp. 1-5, 2018.

[16] N.P.N. Sreedharan, B. Ganesan, R. Raveendran, P. Sarala, B. Dennis, "Grey Wolf optimisation-based feature selection and classification for facial emotion recognition", IET Biometrics, Vol. 7(5), pp. 490-499, 2018.

[17] X. Song, L. Tang, S. Zhao, X. Zhang, L. Li, J. Huang, W. Cai, W., "Grey Wolf Optimizer for parameter estimation in surface waves", Soil Dynamics and Earthquake Engineering, Vol. 75, pp. 147-157, 2015.

[18] P.B. de Moura Oliveira, H. Freire, E.S. Pires, "Grey wolf optimization for PID controller design with prescribed robustness margins”, Soft Computing, Vol. 20(11), pp. 4243-4255, 2016.

[19] S.K. Verma, S. Yadav, S.K. Nagar, "Optimization of fractional order PID controller using grey wolf optimizer", Journal of Control, Automation and Electrical Systems, Vol. 28(3), pp. 314-322, 2017.

[20] M.H. Suid, M.A. Ahmad, R.M.T. Raja Ismail, M.R. Ghazali, A. Irawan, M.Z. Mohd Tumari," An improved sine cosine algorithm for solving optimization problems," Proc. of IEEE Conference on Systems, Process and Control, 2018, to appear.

[21] J.D. Grunnet, M. Soltani, T. Knudsen, M. Kragelund, T. Bak, "Aeolus toolbox for dynamic wind farm model, simulation and control,” Proc. Eur. Wind Energy Conference, pp. 1-10, 2010.

[22] N. Mittal, U. Singh, B.S. Sohi, "Modified grey wolf optimizer for global engineering optimization", Applied Computational Intelligence and Soft Computing, pp. 1-16, 2016.

[23] J.R. Koza, "Genetic programming: on the programming of computers by means of natural selection," MIT Press, Cambridge, 1992.

[24] R. Eberhart, J. Kennedy, “A new optimizer using particle swarm theory,” Proc. of the Sixth International Symposium on Micro Machine and Human Science, pp. 39-43, 1995.

[25] M.A. Meziane, Y. Mouloudi, B. Bouchiba, A. Laoufi, "Impact of inertia weight strategies in particle swarm optimization for solving economic dispatch problem," Indonesian Journal of Electrical Engineering and Computer Science, Vol. 13(1), pp. 377-383, 2019.

\section{BIOGRAPHIES OF AUTHORS}

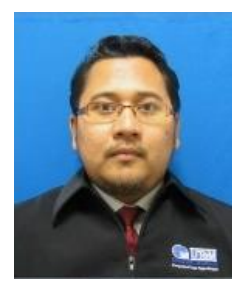

Mohd Zaidi MohdTumari is born at Kuala Lumpur, Malaysia in 1985. He received his first degree in B.Eng. Electrical Mechatronics in 2008 from UniversitiTeknologi Malaysia (UTM) in Johor, Malaysia. In 2010, he received a Master degree in M.Eng. Mechatronics and Automatic Control from UniversitiTeknologi Malaysia (UTM). He has an experience as a research assistant at Robotic Laboratory in UniversitiTeknologi Malaysia (UTM) in 2008, Tutor in UniversitiPendidikan Sultan Idris (2008-2011) and Lecturer in Universiti Malaysia Pahang (2011-2014). Currently, he is a Lecturer in Faculty of Engineering Technology, UniversitiTeknikal Malaysia Melaka (UTeM).As an academician and researcher, he has involved in many research and development in field of mechatronics, control and robotics. He has currently published more than 31 paper works in various International Conference and Journals in the field of mechatronics, control and robotics. He also became a professional member in MBOT, BEM and IEM. His current research interests are optimization, vibration and sway control, liquid slosh system and vehicle steer by wire system.

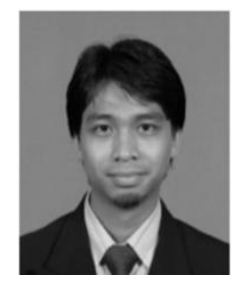

Mohd Helmi Suid received the B.Eng (Hons.) in Electrical and Electronics Engineering from Universiti Teknikal Melaka in 2007. In 2014, he receives M.Sc from School of Electronic and Electrical Engineering, University Sains Malaysia, Penang. He is currently, a lecturer in Faculty of Electrical and Electronics Engineering, University Malaysia Pahang (UMP). His current research interests are digital image processing, vision for robotics application and computational intelligence.

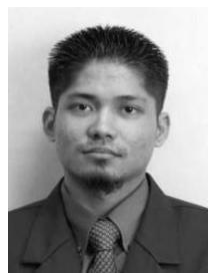

Mohd Ashraf Ahmad received his first degree in B.Eng Electrical Mechatronics and Master Degree in M.Eng Mechatronics and Automatic Control from University of Technology Malaysia (UTM) in 2006 and 2008, respectively. In 2015, he received a Ph.D in Informatics (Systems Science) from Kyoto University. Currently, he is a lecturer in Faculty of Electrical and Electronics Engineering, University Malaysia Pahang (UMP). His current research interests are model-free control, control of mechatronic systems, nonlinear system identification and vibration control. 